\title{
Using age, arterial lactate level and sequential organ failure assessment score in risk stratification of sepsis syndromes
}

\author{
AMR ELMORSI ${ }^{1}$, AMR DAHROUG ${ }^{1}, E N A L F A H M Y^{1}$, ISLAM AHMED \\ 1 Critical Care Medicine Department - Alexandria University, Egypt \\ 2 Clinical Pharmacy and Pharmacy Practice Department - Damanhour University, Egypt
}

The corresponding author:

Amr Elmorsi

Critical Care Medicine Department - Alexandria University, 22 El-Geish Avenue

El-Shatby, Alexandria, EGYPT

Email: Amrabdalla1971icu@gmail.com

\section{ABSTRACT}

Introduction: In low income countries, ICU places are limited and not all sepsis patients will benefit from ICU admission. Stratification is an important step to identify patients who require ICU treatment from patients who can be treated on general ward setting. Improper stratification results in increased length of stay, costs, morbidity and mortality. Objective: The aim of this study was to stratify the risk of mortality in patients with sepsis syndrome using age, arterial lactate level and SOFA score. Methods: In this prospective observational study, 250 patients with sepsis were enrolled and followed up until discharge. They were categorized into 2 groups according to 7-days mortality. Results: SOFA score $(\geq 5)$ was the only good tool $(\mathrm{AUC}=0.722)$ while age $(\geq 65$ years) $(\mathrm{AUC}=0.650)$ and arterial lactate $(\geq 3.25$ $\mathrm{mmol} / \mathrm{L})(0.690)$ were fair tools to predict 7 -days mortality. A new score "ALSOFA score" $(\geq 10)$ was an excellent tool for prediction (AUC $=0.912,95 \% \mathrm{CI}: 0.851$ to $0.940, \mathrm{p}<0.0001)$. It showed an excellent sensitivity (90.9\%) and specificity (85.1\%). Conclusion: In critically ill patients with sepsis syndromes, age, arterial lactate and SOFA score are fair tools of stratification. No single marker/score can be used alone to stratify such patients.

Keywords; Emergency, Critical, Sepsis, SOFA, Arterial Lactate, Stratification

Trial Registration

Alexandria University, IRB No: 00007589 FWA No: 00015712

\section{INTRODUCTION}

Sepsis is officially defined as "a dysregulated host response to an infection, causing life-threatening organ dysfunction". (1) The whole world mortality rates are up to $40 \%$ for sepsis and $70 \%$ for septic shock. (2) Good primary care, source control, early antibiotic and adequate critical care provision are essential for good prognosis in low income countries. (3)

In these low income countries, ICU places are limited and not all sepsis patients will benefit from ICU admission. Stratification is an important step to identify patients who require ICU treatment from patients who can be treated on general ward setting. Improper stratification results in increased length of stay, costs, morbidity and mortality. $(4,5)$

There are multiple tools to stratify patients with sepsis. They include clinical judgement, scoring systems, clinical judgement, or using sepsis categories as defined by the Surviving Sepsis Campaign,(5), which is not as accurate as clinical judgement or scoring systems. (6)

The most applicable scoring systems are the "Predisposition, Infection, Response and Organ dysfunction" (PIRO) score (7), the "Mortality in Emergency Department Sepsis" (MEDS) score (8), the "Mortality In Severe Sepsis in the Emergency Department" (MISSED) score (9) and the wellknown SOFA (10) and qSOFA scores. (5)

The elderly are always at higher risk for sepsis due to multiple comorbidities. In older patients, intact skin and other physiologic reflexes that contribute to the physical defense mechanisms to infections may degrade. Also, implanted devices and surgical procedures may contribute to that. (11)

Serum lactate level is extensively evalu- ated. Arterial lactate is not well studied although it is easy to obtain during routine ABG analysis with low cost. Arterial and serum lactate levels were compared in few studies. Recent studies showed the role of arterial lactate in early diagnosis of sepsis in pediatrics. Adult studies usually advise not to mix their values. (12) The aim of this study was to stratify the risk of mortality in patients with sepsis syndromes using age, arterial lactate level and SOFA score.

\section{METHODS}

After approval of the Medical Ethics Committee of Alexandria Faculty of Medicine, all adult patients who were admitted to the Critical Care Medicine department, Alexandria Main University Hospital for 6 months (from the1st of January 2018 to the 30th of May 2018) with the diagnosis of sepsis (according to 2016 consensus definition (Sepsis-3) using quick SOFA) were assessed for enrollment. Pregnant, trauma and immunocompromised patients (cancer or patients on immunosuppressive drugs) were excluded.

All enrolled patients were subjected directly (at admission) to the followings; complete history taking, physical examination, laboratory investigations and complete sepsis workup. All enrolled patients received the standard treatment for management of sepsis. The protocol of treatment was not changed during the study time. The primary outcome was 7-days mortality.

\section{Statistical Methods}

Data were fed to the computer and analyzed using IBM SPSS software package version 24.0. Qualitative data were described using number and percent. Quantitative data 
were described using range (minimum and maximum), mean, standard deviation and median. Significance of the obtained results was judged at the $5 \%$ level. Chi-square test was used for categorical variables, to compare between different groups. Fisher's Exact or Monte Carlo correction for chisquare was used when more than $20 \%$ of the cells have expected count less than 5 . Student t-test was used for normally quantitative variables. Mann Whitney test was used for abnormally quantitative variables.

\section{RESULTS}

In this prospective observational study, 250 patients were enrolled. Then, they were categorized into 2 groups according to the primary endpoint "7-days mortality". Seventy-seven patients (30.8\%) were died (non-survivors group) and another 173 patients $(69.2 \%)$ were survived (survivors group).

Regarding baseline characteristics, the percentages of males $(52.8 \%)$ and females (47.2\%) were comparable. Pneumonia was the most common suspected source of sepsis $(42 \%)$. Mean age was 63.37 years. Nonsurvivors showed significantly higher mean age (69) than survivors $(60.86)(\mathrm{p}<0.001)$. Non-survivors showed significantly higher SOFA score (7.96) than survivors (5.73) $(p<0.001)$. The median of arterial lactate of all patients was $4 \mathrm{mmol} / \mathrm{L}$. Non-survivors were presented with significantly higher median of arterial lactate (5.0) than survivors (3.2) $(\mathrm{p}<0.001)$. (Table 1)

Overall median ICU LOS was 6 days. Patients with septic shock showed compara-

Table 1. Baseline Characteristics of all enrolled patients

\begin{tabular}{|c|c|c|c|c|c|c|c|}
\hline & \multicolumn{2}{|c|}{$\begin{array}{l}\text { Total } \\
(n=250)\end{array}$} & \multicolumn{2}{|c|}{$\begin{array}{l}\text { Survivors } \\
(\mathrm{n}=173)\end{array}$} & \multicolumn{2}{|c|}{$\begin{array}{l}\text { Non-survivors } \\
(\mathbf{n}=77)\end{array}$} & \multirow[t]{2}{*}{ p value } \\
\hline & No. & $\%$ & No. & $\%$ & No. & $\%$ & \\
\hline Male & 132 & 52.8 & 91 & 52.6 & 41 & 53.24 & 1.000 \\
\hline Female & 118 & 47.2 & 82 & 47.39 & 36 & 46.75 & \\
\hline Age (years) & \multicolumn{2}{|c|}{$63.37 \pm 13.71$} & \multicolumn{2}{|c|}{$60.86 \pm 14.57$} & \multicolumn{2}{|c|}{$69.00 \pm 9.44$} & $<0.001^{\star}$ \\
\hline Hypertension & 85 & 34 & 55 & 31.8 & 30 & 39.0 & 0.166 \\
\hline Diabetes & 85 & 34 & 56 & 32.4 & 29 & 37.7 & 0.470 \\
\hline Hepatic & 45 & 18 & 30 & 17.3 & 15 & 19.5 & 0.723 \\
\hline Stroke & 23 & 9.2 & 15 & 8.7 & 8 & 10.4 & 0.643 \\
\hline Renal & 42 & 16.8 & 32 & 18.5 & 10 & 13.0 & 0.360 \\
\hline Ischemic & 21 & 8.4 & 13 & 7.5 & 8 & 10.4 & 0.465 \\
\hline Chronic Afib & 38 & 15.2 & 22 & 12.7 & 16 & 20.8 & 0.126 \\
\hline COPD & 7 & 2.8 & 4 & 2.3 & 3 & 3.9 & 0.680 \\
\hline Miscellaneous & 5 & 2 & 2 & 1.2 & 3 & 3.9 & 0.172 \\
\hline SOFA score & \multicolumn{2}{|c|}{$6.42 \pm 2.852$} & \multicolumn{2}{|c|}{$5.73 \pm 2.554$} & \multicolumn{2}{|c|}{$7.96 \pm 2.895$} & $<0.001^{\star}$ \\
\hline APACHE II & \multicolumn{2}{|c|}{$23.04 \pm 6.40$} & \multicolumn{2}{|c|}{$20.90 \pm 5.65$} & \multicolumn{2}{|c|}{$27.86 \pm 5.33$} & $<0.001^{\star}$ \\
\hline Cellulitis & 14 & 5.6 & 9 & 5.2 & 5 & 6.5 & 0.767 \\
\hline Pneumonia & 105 & 42 & 79 & 45.7 & 26 & 33.8 & 0.096 \\
\hline UTI & 41 & 16.4 & 28 & 16.2 & 13 & 16.9 & 1.000 \\
\hline DFI & 11 & 4.4 & 6 & 3.5 & 5 & 6.5 & 0.321 \\
\hline SBP & 23 & 9.2 & 13 & 7.5 & 10 & 13.0 & 1.000 \\
\hline Abdomen & 12 & 4.8 & 7 & 4.1 & 5 & 6.5 & 0.705 \\
\hline CRBSI & 21 & 8.4 & 10 & 5.8 & 11 & 14.3 & 0.054 \\
\hline Mixed & 23 & 9.2 & 10 & 5.8 & 13 & 16.9 & 0.236 \\
\hline $\mathrm{SBP}(\mathrm{mmHg})$ & \multicolumn{2}{|c|}{$73.68 \pm 31.42$} & \multicolumn{2}{|c|}{$82.66 \pm 27.59$} & \multicolumn{2}{|c|}{$53.51 \pm 30.25$} & $<0.001^{*}$ \\
\hline $\mathrm{DBP}(\mathrm{mmHg})$ & \multicolumn{2}{|c|}{$43.98 \pm 22.0$} & \multicolumn{2}{|c|}{$50.20 \pm 20.10$} & \multicolumn{2}{|c|}{$30.0 \pm 19.76$} & $<0.001^{*}$ \\
\hline HR (beats/min) & \multicolumn{2}{|c|}{$114.1 \pm 20.39$} & \multicolumn{2}{|c|}{$110.8 \pm 20.86$} & \multicolumn{2}{|c|}{$121.7 \pm 17.14$} & $<0.001^{*}$ \\
\hline Temp. $\left({ }^{\circ} \mathrm{C}\right)$ & \multicolumn{2}{|c|}{$38.11 \pm 0.940$} & \multicolumn{2}{|c|}{$38.22 \pm 0.911$} & \multicolumn{2}{|c|}{$37.87 \pm 0.969$} & $0.036^{*}$ \\
\hline RR (breath/min) & \multicolumn{2}{|c|}{$26.96 \pm 7.221$} & \multicolumn{2}{|c|}{$25.88 \pm 6.42$} & 29.38 & & $0.001^{*}$ \\
\hline WBCs $\times 109 / \mathrm{L}$ & 16.7 & & 16.18 & & $17.9=$ & & 0.207 \\
\hline CRP (mg/L) & 109.8 & & 99.4 & & 133.3 & & $<0.001^{*}$ \\
\hline Urea (mg/dL) & 104. & & 95.98 & & 123.6 & & $0.001^{\star}$ \\
\hline S.Cr (mg/dL) & 2.46 & & 2.35 & & $2.71=$ & & $0.003^{*}$ \\
\hline $24 \mathrm{~h}-\mathrm{UOP}(\mathrm{mL} / \mathrm{hr})$ & 39.95 & & 44.2 & & 30.26 & & $<0.001^{\star}$ \\
\hline GCS & 12.42 & & 12.6 & & 11.82 & & $0.001^{*}$ \\
\hline Lactate $(\mathrm{mmol} / \mathrm{L})$ & 4.76 & & 4.18 & & $4.76=$ & & $<0.001^{\star}$ \\
\hline
\end{tabular}

COPD: chronic obstructive pulmonary disease, UTI: urinary tract infection, DFI: Diabetic Foot Infection, SBP: Spontaneous Bacterial Peritonitis, CRBSI: Catheter-Related Blood Stream Infection. Abdomen sources includes perforated gut and cholangitis. SBP: Systolic Blood Pressure, DBP: Diastolic Blood Pressure, HR: Heart Rate, RR: Respiratory rate, Temp: surface body Temperature. S.Cr: Serum Creatinine, UOP: Urine Output, GCS: Glasgow Coma Scale. ${ }^{*} \mathrm{p}$ is significant when $\mathrm{p} \leq 0.05$ 
Table 2. Agreement (sensitivity, specificity) of study stratification tools to predict 7-days mortality

\begin{tabular}{llllllllll}
\hline & AUC & p value & 95\% C.I & & Cut off & Sensitivity & Specificity & PPV & NPV \\
\hline & & & LL & UL & & & & & \\
Age (years) & 0.650 & $<0.0001^{*}$ & 0.578 & 0.721 & $\geq 65$ & 84.4 & 37.8 & 37.6 & 84.4 \\
\hline $\begin{array}{l}\text { Arterial } \\
\text { lactate(mmol/L) }\end{array}$ & 0.690 & $<0.0001^{*}$ & 0.617 & 0.763 & $\geq 3.25$ & 80.5 & 51.4 & 42.5 & 85.6 \\
\hline SOFA Score & 0.722 & $<0.0001^{*}$ & 0.653 & 0.791 & $\geq 5$ & 83.1 & 53.8 & 44.4 & 87.8 \\
\hline ALSOFA score & 0.912 & $<0.0001^{*}$ & 0.851 & 0.940 & $\geq 10$ & 90.9 & 85.1 & 53.4 & 94.1 \\
\hline
\end{tabular}

AUC: Area Under Curve, C.I: Confidence Interval, PPV: Positive Predictive Value, NPV: Negative Predictive Value

Table 3. The calculation of ALSOFA score

\begin{tabular}{llllll}
\hline Points & $\mathbf{1}$ & $\mathbf{2}$ & $\mathbf{3}$ & $\mathbf{4}$ & $\mathbf{5}$ \\
\hline Age (years) & 18 to $<35$ & 35 to $<50$ & 50 to $<55$ & 55 to $<60$ & $\geq 60$ \\
\hline Arterial lactate $(\mathrm{mmol} / \mathrm{L})$ & $<3$ & $3:<4$ & $4:<5$ & $5:<6$ & $\geq 6$ \\
\hline SOFA Score & $<2$ & $2-3$ & $4-5$ & $6-7$ & $>7$ \\
\hline
\end{tabular}

Table 4. ALSOFA score in prediction of mortality

\begin{tabular}{lll}
\hline ALSOFA score & 7-days mortality & 28-days mortality \\
\hline $13-15$ & $66-90 \%$ & $87-95 \%$ \\
\hline $10-12$ & $15-47 \%$ & $59-80 \%$ \\
\hline$\otimes 10$ & $<15 \%$ & $<59 \%$ \\
\hline p value & $<0.0001^{*}$ & $<0.0001^{*}$ \\
\hline Exp $(\beta)$ & 2.191 & 1.667 \\
\hline $95 \% \mathrm{CI}$ & $1.827: 2.627$ & $1.443: 1.926$ \\
\hline
\end{tabular}

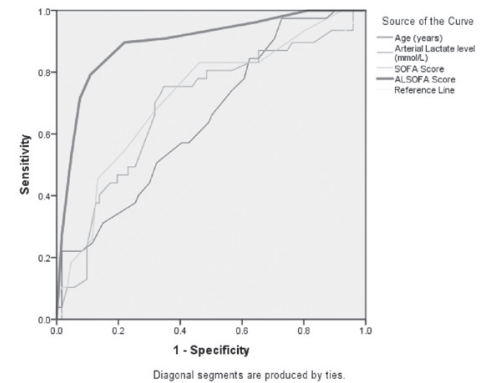

Figure 1. Receiver Operating Characteristics (ROC) curve of study stratification tools to predict 7-days mortality

ble median of LOS (5 days) with patients with sepsis $(8)(p=0.081)$. Overall 28 -days mortality rate was $59.2 \%$. Patients with septic shock showed comparable mortality $(66.7 \%)$ with patients with sepsis $(55.2 \%)$ $(\mathrm{p}=0.105)$.

After plotting the receiver operating characteristics (ROC) curve for the study stratification tools to predict 7-days mortality of all patients (Table 2) (Figure $1)$, SOFA score $(\geq 5)$ was the only good tool (AUC $=0.722)$ while age $(\geq 65$ years) $(\mathrm{AUC}=0.650)$ and arterial lactate $(\geq 3.25$ $\mathrm{mmol} / \mathrm{L})(0.690)$ were fair tools to predict

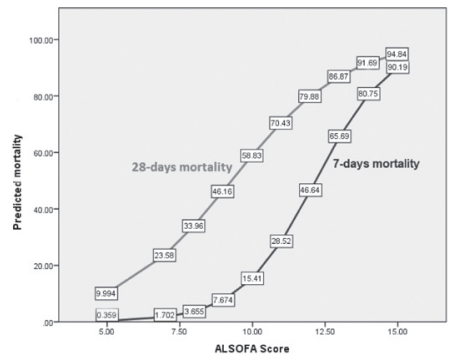

Figure 2. The prediction of mortality using ALSOFA score

7-days mortality. A new score was implemented using these 3 tools. This score ranges from 3 to 15 points. It was arbitrarily mentioned as "ALSOFA score: Age, Lactate and SOFA score". It was calculated for all enrolled patients. (Table 3 )

ALSOFA score $(\geq 10)$ was an excellent tool to predict 7-days mortality (AUC=0.912). After logistic regression analysis, as ALSOFA score increases by one point the odds ratio (OR) of 7-days mortality increases by 2.191 (68.66\% increased risk) $(\mathrm{p}<0.0001)$. (Table 4) (Figure 2)

\section{DISCUSSION}

In this study, age $\geq 65$ years was a fair tool to predict 7-days mortality $(\mathrm{AUC}=0.650$, 95\% CI: 0.578 to $0.721, \mathrm{p}<0.0001)$. De Groot et al., investigated whether the prognostic utility of the most common severity scores were appropriate for risk stratification of older ( $\geq 70$ years) septic patients ( $\geq 70$ years). In-hospital mortality was 9.5\% (95\%CI: 7.4 to 11.5 ) in older patients, and $4.6 \%$ (95\%CI: 3.6 to 5.7 ) in younger patients. In older patients, disease severity scores were associated poorly with mortality (AUC $=0.56$ to 0.64$)$. (13)

Warmerdam et al., showed that mortality in patients with infections was 9.2\% (95\%CI: 7.3 to 11.2 ) in patients $\geq 70$, twice as high as the $4.6 \%(3.6-5.6)$ in patients $<70$ years. (14) Ginde et al., showed that older adults ( $\geq 65$ years) with severe sepsis, compared with younger adults, had modestly higher rate of mortality (24\% vs $16 \%$ ). (15) Yang et al., showed that age (OR, 8.46; aged 85 years and older versus aged $18-54$ years old) was significant and independent predictor of hospital mortality. (16) Martin et al., showed that age was an independent predictor of mortality in an adjusted multi- 
variable regression (OR, 2.26; 95\%CI: 2.17 to 2.36 ). Elderly sepsis patients died earlier during hospitalization. (17)

In this study, arterial lactate level $\geq 3.25$ $\mathrm{mmol} / \mathrm{L}$ was a fair tool to predict 7-days mortality (AUC $=0.690,95 \%$ CI: 0.617 to $0.763, \mathrm{p}<0.0001)$. In critical care units, lactate is routinely used for risk stratification. Whether venous or arterial lactate measured on blood gas is interchangeable is not known. To our knowledge, no studies was conducted to stratify sepsis patients according to their initial arterial lactate levels. But, Paquet et al., showed that both methods presented similar performances for the prediction of poor prognosis (AUC= 0.67). (18)

Recently, Theerawit et al., showed that arterial and venous lactate levels were strongly correlated $(\mathrm{r}=0.934, \mathrm{p}<0.0001$, r2 = .873). (19) Also, Datta et al., showed that the mean difference between them was $0.4 \mathrm{mmol} / \mathrm{L}$ [ $95 \% \mathrm{CI}: 0.37$ to 0.45 ], with 95\% limits of agreement from -0.4 (95\%CI: -0.45 to -0.32 ) to 1.2 (95\%CI: $1.14-1.27$ ). Also, venous level less than $2 \mathrm{mmol} / \mathrm{l}$ was predictive of an arterial level less than $2 \mathrm{mmol} / \mathrm{l}$. (20)

In Diao et al. study, arterial lactate $\otimes 1.7$ $\mathrm{mmol} / \mathrm{L}$ was a good tool to predict mortality $(\mathrm{AUC}=0.805)$ with a good sensitivity (79.1\%). (21)

In this study, SOFA score $\geq 5$ was a good tool to predict 7-days mortality (AUC= $0.722,95 \%$ CI: 0.653 to $0.791, \mathrm{p}<0.0001)$. Innocenti et al., showed that SOFA score was associated with a moderate prognostic stratification ability. (22)

In contrast to these findings, Garcia-Villalba et al., showed that SOFA score was an inadequate prognostic tool in patients at low risk of organ damage. Results showed that other clinical and analytical variables are required to improve the prognostic utility of SOFA score. (23)

Macdonald et al., (2014) showed that PIRO score (AUC $=0.86(95 \% \mathrm{CI}$ : 0.80 to 0.92$)$ ) and MEDS (AUC=0.81 (95\%CI: 0.74 to $0.88)$ ) were better predictor of mortality than SOFA score (AUC=0.78 (95\%CI: 0.71 to 0.87$))(24)$

In this study, the new stratification tool was arbitrarily mentioned ALSOFA score. ALSOFA score $\geq 10$ was an excellent tool to predict 7-days mortality (AUC $=0.912$ (95\%CI: 0.851 to 0.940$)) \quad(\mathrm{p}<0.0001)$. It showed excellent sensitivity (90.9\%), specificity (85.1\%), PPV (53.4\%) and NPV (4.1\%).

Bewersdorf et al., showed that a new score (The SPEED, Sepsis Patient Evaluation in the Emergency Department) was a very good tool to predict 28-day mortality in septic patients $(\mathrm{AUC}=0.81$ (95\%CI: 0.75 to 0.86$)$ ) in the derivation and ( $\mathrm{AUC}=0.81$ ( $95 \%$ CI: 0.73 to 0.90$)$ ) in the validation set. (25)

\section{LIMITATIONS}

This study has some limitations, as small sample size and monocentric design. The study design is liable for multiple con- founding factors. The primary outcome is 7-days all-cause mortality. We acknowledge that these finding cannot be directly extrapolated to other health care facilities.

\section{CONCLUSION}

In critically ill patients with sepsis syndromes, age, arterial lactate and SOFA score are fair tools of stratification. No single marker/score can be used alone to stratify such patients. Further larger studies should be conducted validate new scores. Simple, available and cheap markers should be used in such scores otherwise scoring systems should not be used as hard criteria for ICU admission.

\section{DECLARATIONS}

Acknowledgements

Not applicable.

\section{Funding}

No funding to declare.

Availability of data and materials

Please contact author for any data requests.

\section{Competing interests}

The author declares that there are no competing interests

\section{REFERENCES}

1. Singer M, Deutschman CS, Seymour CW, Shankar-Hari M, Annane D, Bauer M, et al. The third international consensus definitions for sepsis and septic shock (Sepsis-3). JAMA 2016;315(8):801-10.

2. Jawad I, Lukšić I, Rafnsson SB. Assessing available information on the burden of sepsis: global estimates of incidence, prevalence and mortality. J Glob Health 2012;2(1):1-4.

3. Fleischmann C, Scherag A, Adhikari NK, Hartog CS, Tsaganos T, Schlattmann P, et al. Assessment of global incidence and mortality of hospital-treated sepsis. Current estimates and limitations. Am J Respir Crit Care Med 2016;193(3):259-72.

4. Kennedy M, Joyce N, Howell MD, Mottley JL, Shapiro NI. Identifying infected emergency department patients admitted to the hospital ward at risk of clinical deterioration and intensive care unit transfer. Acad Emerg Med 2010;17(10):1080-5.

5. Singer M, Deutschman CS, Seymour CW, Shankar-Hari M, Annane D, Bauer M, et al. The Third International Consensus Definitions for Sepsis and Septic Shock (Sepsis-3). JAMA2016;315(8):801-10.

6. de Groot B, Lameijer J, de Deckere ER, Vis A. The prognostic performance of the predisposition, infection, response and organ failure (PIRO) classification in high-risk and low-risk emergency department sepsis populations: comparison with clinical judgement and sepsis category. Emerg Med J 2014;31(4):292-300.

7. Rubulotta F, Marshall JC, Ramsay G, Nelson D, Levy M, Williams M. Predisposition, insult/infection, response, and organ dysfunction: A new model for staging severe sepsis. Crit Care Med 2009;37(4):1329-35.

8. Shapiro NI, Wolfe RE, Moore RB, Smith E, Burdick E, Bates DW. Mortality in Emergency Department Sepsis (MEDS) score: a prospectively derived and validated clinical prediction rule. Crit Care Med 2003;31(3):670-5.

9. Sivayoham N, Rhodes A, Cecconi M. The MISSED score, a new scoring system to predict Mortality In Severe Sepsis in the Emergency Department: a derivation and validation study. Eur J Emerg Med.2014;21(1):30-6.

10. Vincent J-L, Moreno R, Takala J, Willatts S, De Mendonça A, Bruining H, et al. The SOFA (Sepsis-related Organ Failure Assessment) score to describe organ dysfunction/failure. On behalf of the Working Group on Sepsis-Related Problems of the European Society of Intensive Care Medicine. Intensive Care Med 1996 Jul;22(7):707-10. 
11. Opal SM, Girard TD, Ely EW. The immunopathogenesis of sepsis in elderly patients. Clin Infect Dis 2005;41 Suppl 7:S504-12.

12. Schlapbach LJ, MacLaren G, Straney L. Venous vs arterial lactate and 30-day mortality in pediatric sepsis. JAMA Pediatr 2017;171(8):813-.

13. de Groot B, Stolwijk F, Warmerdam M, Lucke JA, Singh GK, Abbas M, et al. The most commonly used disease severity scores are inappropriate for risk stratification of older emergency department sepsis patients: an observational multi-centre study. Scand J Trauma Resusc Emerg Med 2017;25:91.

14. Warmerdam M, Stolwijk F, Boogert A, Sharma M, Tetteroo L, Lucke J, et al. Initial disease severity and quality of care of emergency department sepsis patients who are older or younger than 70 years of age. PloS one. 2017;12(9):e0185214.

15. Ginde AA, Moss M, Shapiro NI, Schwartz RS. Impact of older age and nursing home residence on clinical outcomes of US emergency department visits for severe sepsis. J Crit Care 2013;28(5):606-11.

16. Yang Y, Yang KS, Hsann YM, Lim V, Ong BC. The effect of comorbidity and age on hospital mortality and length of stay in patients with sepsis. J Crit Care 2010;25(3):398-405.

17. Martin GS, Mannino DM, Moss M. The effect of age on the development and outcome of adult sepsis. Crit Care Med 2006;34(1):1521.

18. Paquet AL, Valli V, Philippon AL, Devilliers C, Bloom B, Hausfater P, et al. Agreement between arterial and venous lactate in emergency department patients: a prospective study of 157 consecutive patients. Eur J Emerg Med 2018;25(2):92-6.

19.19. Theerawit P, Na Petvicharn C, Tangsujaritvijit V, Sutherasan Y. The correlation between arterial lactate and venous lactate in patients with sepsis and septic shock. J Intensive Care Med2018;33(2):116-20.

20. Datta D, Grahamslaw J, Gray AJ, Graham C, Walker CA. Lactate - Arterial and venous agreement in sepsis: a prospective observational study. Eur J Emerg Med 2018;25(2):85-91.

21. Diao MY, Wang T, Cui YL, Lin ZF. [Prognostic value of arterial lactate content combined with base excess in patients with sepsis: a retrospective study]. Zhonghua wei zhong bing ji jiu yi xue. 2013;25(4):211-4.

22. Innocenti F, Tozzi C, Donnini C, De Villa E, Conti A, Zanobetti M, et al. SOFA score in septic patients: incremental prognostic value over age, comorbidities, and parameters of sepsis severity. Intern Emerg Med2018;13(3):405-12.

23. Garcia-Villalba E, Cano-Sanchez A, Alcaraz-Garcia A, Cinesi-Gomez C, Pinera-Salmeron P, Marin I, et al. [Nomogram to predict a poor outcome in emergency patients with sepsis and at low risk of organ damage according to Sepsis-related Organ Failure Assessment (SOFA)]. Emergencias2017;29(2):81-6.

24. Macdonald SP, Arendts G, Fatovich DM, Brown SG. Comparison of PIRO, SOFA, and MEDS scores for predicting mortality in emergency department patients with severe sepsis and septic shock. Acad Emerg Med2014;21(11):1257-63.

25. Bewersdorf JP, Hautmann O, Kofink D, Abdul Khalil A, Zainal Abidin I, Loch A. The SPEED (sepsis patient evaluation in the emergency department) score: a risk stratification and outcome prediction tool. Eur J Emerg Med 2017;24(3):170-5. 